080 was also able to reverse HLA-G-mediated suppression of $\mathrm{ILT}^{+} \mathrm{CD}^{+} \mathrm{T}$ cells as assessed by degranulation and proinflammatory cytokine secretion. Notably, mice with disseminated tumors had extended median survival when treated with a single dose of TTX-080.

Conclusions TTX-080 reverses HLA-G-mediated suppression of $\mathrm{ILT}_{2}{ }^{+}$and $\mathrm{ILT}^{+}{ }^{+}$immune cells that are found within the tumor microenvironment. Blockade of HLA-G using TTX-080 therefore has the potential to reverse broad immune suppression in patients with advanced solid tumors by reinvigorating $\mathrm{CD}^{+} \mathrm{T}$ cells, enhancing NK cytolytic activity, and increasing macrophage phagocytosis.

http://dx.doi.org/10.1136/jitc-2020-SITC2020.0698

\section{A DIFFERENTIATED ANTI-OX40 AGONIST BGB-A445 DOES NOT BLOCK OX40-OX40L INTERACTION AND REVEALS REMARKABLE ANTI-TUMOR EFFICACY IN PRECLINICAL MODELS}

Ye Liu*, Beibei Jiang, Tong Zhang, Zuobai Wang, Yingcai Feng, Haiying Li, Wenfeng Gong, Xing Wang, Yajuan Gao, Xiaosui Zhou, Bo Zhang, Yuan Hong, Jing Wang, Zilin Wang, Hongjia Hou, Hanzi Sun, Xiaomin Song, Kang Li, Xuesong Liu. BeGene (Beijing) Co., Ltd., Beijing, China

Background OX40 is a member of the tumor necrosis factor receptor super family (TNFRSF) primarily expressed on activated $\mathrm{CD} 4+$ and $\mathrm{CD} 8+\mathrm{T}$ cells, as well as natural killer (NK) $\mathrm{T}$ and NK cells. It is an immune costimulatory receptor which

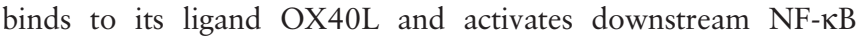
pathway to induce immune cell activation, proliferation, and survival. ${ }^{1-3}$ Current agonistic anti-OX40 antibodies in clinic, which are mostly ligand-competitive antibodies, showed limited clinical responses, mainly at lower doses. Blockade of OX40OX40L interaction might limit the efficacy of these ligandcompetitive antibodies at higher doses, as OX40-OX40L interaction is essential for enhancing effective anti-tumor immunity. Here we report pre-clinical data of BGB-A445, which is a ligand non-blocking agonistic anti-OX40 humanized antibody.

Methods Cell-based flow cytometry assay was established to determine whether BGB-A445 interferes with OX40-OX40L interaction. Co-crystal structure of OX40/BGB-A445 Fab was solved to study the molecular binding mechanism. A mixed lymphocyte reaction (MLR) assay was set up to investigate the ability of BGB-A445 to activate CD4+ T-cells. The anti-tumor efficacy of BGB-A445 was evaluated in MC38 colon cancer and CT26WT colon cancer models either as a single agent or in combination with anti-PD-1 antibody.

Results The flow cytometry study showed that BGB-A445 did not interfere with the binding of OX40 to OX40L even at high concentrations. In contrast, MOXR0916, an anti-OX40 agonistic antibody developed by Genentech, completely blocked OX40 binding to OX40L. Additionally, the co-crystal structure of OX40/BGB-A445 Fab complex indicated that BGB-A445 interacts with the CRD4 region of OX40 which is distant from OX40L binding region. In the MLR assay, combined with an anti-PD-1 antibody, BGB-A445 co-stimulated CD4+ T-cells to secrete IL-2 dose-dependently, while MOXR0916 did not. In the MC38 colon cancer model in human OX40 knock-in mice, BGB-A445 demonstrated remarkable anti-tumor efficacy in a dose-dependent manner, while MOXR0916 showed a 'hook effect' in the same setting. In addition, BGB-A445 exhibited significant anti-tumor activity in the PAN02 pancreatic model which is resistant to anti-PD-1 treatment. Besides, BGB-A445 revealed significant combination effects with anti-PD-1 therapy in both MC38 and CT26WT models.

Conclusions In conclusion, differentiated from current clinical stage anti-OX40 antibodies, BGB-A445 is an agonistic antibody that does not block the OX40-OX40L interaction. Both in vitro and in vivo results demonstrated that BGB-A445 has remarkable immune stimulating effect and anti-tumor efficacy either as a single agent or in combination with anti-PD-1 therapy, thus warranting further clinical investigation.

\section{REFERENCES}

1. Croft M. Control of immunity by the TNFR-related molecule OX40 (CD134). Annu Rev Immunol 2010;28:57-78.

2. Gramaglia I, et al. Ox-40 ligand: a potent costimulatory molecule for sustaining primary CD4 T cell responses. J Immunol 1998:161:6510-6517.

3. Song J, So T, Croft M. Activation of NF-kappaB1 by OX40 contributes to antigendriven T cell expansion and survival. J Immunol 2008;180:7240-7248.

http://dx.doi.org/10.1136/jitc-2020-SITC2020.0699

\section{EPHA2/CD137 BICYCLE TUMOR-TARGETED IMMUNE CELL AGONISTS (TICAS ${ }^{\text {TM}}$ ) INDUCE TUMOR REGRESSIONS, IMMUNOGENIC MEMORY, AND REPROGRAMMING OF THE TUMOR IMMUNE MICROENVIRONMENT}

Kristen Hurov*, Johanna lahdenranta, Gemma Mudd, Punit Upadhyaya, Elizabeth Repash, Jun Ma, Julia Kristensson, Marianna Kleyman, Jessica Kublin, Liuhong Chen, Eric Haines, Sailaja Battula, Kevin McDonnell, Philip Brandish, Nicholas Keen. Bicycle Therapeutics, Lexington, MA, USA

Background Despite compelling preclinical data, agonistic antiCD137 antibodies have been hampered by failure to delineate hepatotoxicity from efficacy in clinical studies. ${ }^{12}$ A new generation of both systemic and targeted CD137 agonists that are now entering clinical development rely on biologic agents with suboptimal properties for CD137 agonism due to their relatively large sizes and long circulating half-lives. ${ }^{3-5}$ These properties may limit their tissue penetration and cause sustained agonism resulting in overstimulation and activationinduced cell death of lymphocytes due to continuous exposure.BCY12491 is a tumor-targeted immune cell agonist $\left(\mathrm{TICA}^{\mathrm{TM}}\right.$ ) that exemplifies a new class of fully synthetic immunomodulators with constrained bicyclic peptides (Bicycles ${ }^{\circledR}$ ) targeting a tumor antigen and a co-stimulatory molecule. We developed this new class of synthetic molecules with antibody-like affinities and target selectivity to circumvent the beforementioned barriers to optimal targeted CD137 agonistic therapeutics. BCY12491 (EphA2/CD137 TICA) is designed to deliver a highly potent CD137 agonist to EphA2 overexpressing tumor tissue with an intermittent dosing schedule maximizing anti-tumor activity while circumventing the need for continuous systemic exposure.

Methods BCY12491 bioactivity was assessed in vitro using a CD137 reporter assay and by measuring cytokine production from primary human PBMC/tumor cell co-cultures. BCY12491 in vivo activity was determined in huCD137-syngeneic tumor models by measuring tumor growth kinetics and using tumor immune cell and transcriptional profiling by FACS, IHC, and Nanostring.

Results BCY12491 engages EphA2 and CD137 with high affinity resulting in picomolar potency in co-culture assays consisting of EphA2-expressing tumor cell lines and CD137expressing Jurkat NF-kappaB-luciferase reporter cells. 
Moreover, BCY12491 caused EphA2-dependent CD137 agonism in primary human PBMCs co-cultured with tumor cells with varied levels of EphA2 expression. Treatment of MC38 tumors in immunocompetent mice with BCY12491 leads to a profound reprogramming of the tumor immune microenvironment including increased $\mathrm{T}$ cell infiltration and stimulation of NF-kappaB signaling, costimulatory signaling, cytotoxicity and cytokine/chemokine signaling functional pathways. BCY12491 treatment leads to $\mathrm{MC} 38$ tumor regressions, complete responses, and immunogenic memory without continuous drug exposure in the periphery. This anti-tumor activity is dependent on CD8 + T cells, but not on NK $1.1+$ cells.

Conclusions BCY12491 is a potent EphA2-dependent CD137 agonist with optimal target binding, pharmacologic, and pharmacokinetic properties that enable anti-tumor TME remodeling and complete responses in vivo with intermittent dosing. This work unleashes a new and tractable avenue to testing a novel class of therapeutic CD137 agonists in humans for the treatment of cancer.

Ethics Approval The care and use of animals were reviewed and approved by the Institutional Animal Care and Use Committee (IACUC) of WuXi AppTec and conducted in accordance with the regulations of the Association for Assessment and Accreditation of Laboratory Animal Care (AAALAC).

\section{REFERENCES}

1. Segal NH, Logan TF, Hodi FS, et al. Results from an integrated safety analysis of urelumab, an agonist anti-CD137 monoclonal antibody. Clin Cancer Res 2017;23 (8):1929-1936.

2. Chester C, Sanmamed MF, Wang J, Melero I. Immunotherapy targeting 4-1BB: mechanistic rationale, clinical results, and future strategies. Blood 2018;131(1): 49-57.

3. Hinner MJ, Aiba RSB, Jaquin TJ, et al. Tumor-Localized Costimulatory T-Cell Engagement by the 4-1BB/HER2 Bispecific Antibody-Anticalin Fusion PRS-343. Clin Cancer Res. 2019;25(19):5878-5889.

4. Claus C, Ferrara, C, Xu W, et al. Tumor-targeted 4-1BB agonists for combination with T cell bispecific antibodies as off-the-shelf therapy. Sci Transl Med 2019;11 (496): eaav5989.

5. Eskiocak U, Guzman W, Wolf $B$, et al. Differentiated agonistic antibody targeting CD137 eradicates large tumors without hepatotoxicity. JCI Insight 2020;5(5): e133647.

http://dx.doi.org/10.1136/jitc-2020-SITC2020.0700

\section{NANOPARTICULATE MONOBENZONE (MBEH) AS A POTENTIAL DRUG CANDIDATE FOR MELANOMA}

${ }^{1}$ Dinesh Jaishankar*, 'Steven Henning, ${ }^{1}$ Anqi Zhang, ${ }^{2}$ Basar Bilgicer, ${ }^{1}$ SonBinh Nhuyen, ${ }^{1}$ I Caroline Le Poole. 'Northwestern University, Chicago, IL, USA; '2University of Notre Dame, South Bend, IN, USA

Background Monobenzone (MBEH) is a skin depigmenting agent FDA-approved for topical applications. ${ }^{1}$ It specifically interacts with tyrosinase, ${ }^{2}$ a key enzyme in melanogenesis, to form reactive quinones that are toxic to pigmented cells, including melanoma cells. As melanoma cells express abundant tyrosinase activity, ${ }^{3}$ repurposing $\mathrm{MBEH}$ to target melanoma cells might serve as a treatment strategy. Furthermore, quinones can haptenize tyrosinase ${ }^{4}$ supporting neo-antigen formation. ${ }^{5}$ Modified tumor antigens then initiate an immune cascade, engaging $\mathrm{T}$ cells to target tumor cells. This biphasic effect of $\mathrm{MBEH}$ makes it a suitable candidate to target melanoma. Although topical treatment of MBEH can suppress subcutaneous melanoma growth in vivo, ${ }^{6}$ systemic administration of the drug was toxic, ${ }^{7}$ limiting the application of $\mathrm{MBEH}$ for metastatic disease. To overcome this limitation, we encapsulated $\mathrm{MBEH}$ and its derivatives into nanoscale liposomes $(\sim 100 \mathrm{~nm})$ and evaluated its anti-tumor efficacy.

Methods Liposomes were prepared ${ }^{8}$ and MBEH was loaded into the liposomes. Loading was evaluated using mass spectroscopy and nuclear magnetic resonance (NMR) spectroscopy. In vitro cytotoxicity of liposomal MBEH to mouse and human melanoma cell lines was evaluated by MTT assays. Meanwhile, in vivo trafficking of fluorescent liposomes to B16-F10 tumors and vital organs was evaluated in tissue homogenates by flow cytometry. The anti-tumor effects of liposomal MBEH towards subcutaneously injected B16-F10 melanoma cells were evaluated in C57BL/6 mice ( $\mathrm{n}=4$ per group) over time.

Results Mass spectroscopy and NMR data revealed that MBEH was encapsulated into the liposomes at $2.3 \% \mathrm{~mol}$ $\mathrm{MBEH}$ per liposome. Liposomal MBEH was toxic to both mouse and human melanoma cells with lower half maximal inhibitory concentration (IC50) values in B16-F10 and A375 and higher IC50 values in 888-A2 and 624.38 cells. In vivo trafficking in mice revealed $\sim 55 \%$ uptake of liposomal fluorescence by the tumor. In contrast to empty liposomes (mean tumor volume on day 21: $467.5 \mathrm{~mm}^{3}$ ), MBEH loaded liposomes significantly $(\mathrm{P}<0.0001)$ reduced $\mathrm{B} 16-\mathrm{F} 10$ tumor growth mice (mean tumor volume on day 21: $107 \mathrm{~mm}^{3}$ ). Skin depigmentation was not observed over the 21 day period of follow up after liposomal MBEH treatment, supporting that liposomal MBEH can be safely administered.

Conclusions We provide a proof-of-concept to use nanoparticulate $\mathrm{MBEH}$ to target the melanogenic pathway in melanoma. A detailed study of $\mathrm{MBEH}$ loading, nanoparticle stability, and tumor infiltrating lymphocyte can further establish nanoparticulate $\mathrm{MBEH}$ as a potential drug candidate for melanoma.

Acknowledgements This work was supported by a grant from the Sherman Fairchild Foundation.

\section{REFERENCES}

1. Bolognia JL, Lapia K, and Somma S, Depigmentation therapy. Dermatologic Therapy 2001. 14(1): p. 29-34.

2. McGuire J and Hendee J, Biochemical Basis for Depigmentation of Skin by Phenolic Germicides. Journal of Investigative Dermatology 1971. 57(4): p. 256-261.

3. Michaeli $Y$, et al., Melanoma cells present high levels of HLA-A2-tyrosinase in association with instability and aberrant intracellular processing of tyrosinase. European Journal of Immunology, 2012. 42(4): p. 842-850.

4. Manini $P$, et al., A reactive ortho-quinone generated by tyrosinase-catalyzed oxidation of the skin depigmenting agent monobenzone: self-coupling and thiol-conjugation reactions and possible implications for melanocyte toxicity. Chem Res Toxicol, 2009. 22(8): p. 1398-405.

5. 5. Westerhof $W$, et al., The haptenation theory of vitiligo and melanoma rejection: a close-up. Experimental Dermatology 2011. 20(2): p. 92-96.

6. Hariharan V, et al., Topical application of bleaching phenols; in-vivo studies and mechanism of action relevant to melanoma treatment. Melanoma Res, 2011. 21 (2): p. 115-26.

7. Kelly KH, Bierman $H R$, and Shimkin MB, Negative effects of oral monobenzyl ether of hydroquinone in malignant melanoma in man. proceedings of the society for experimental Biology and Medicine 1952. 79(4): p. 589-590.

8. Stefanick JF, et al., A systematic analysis of peptide linker length and liposomal polyethylene glycol coating on cellular uptake of peptide-targeted liposomes. ACS Nano 2013. 7(4): p. 2935-2947.

http://dx.doi.org/10.1136/jitc-2020-SITC2020.0701 